UWThPh-2006-22

\title{
Predictions of an $A_{4}$ model with a five-parameter neutrino mass matrix
}

\author{
L. Lavoura ${ }^{(1) \ddagger}$ and H. Kühböck ${ }^{(2) \S}$ \\ (1) Universidade Técnica de Lisboa and Centro de Física Teórica de Partículas \\ Instituto Superior Técnico, 1049-001 Lisboa, Portugal \\ (2) \\ Institut für Theoretische Physik, Universität Wien \\ Boltzmanngasse 5, A-1090 Wien, Austria
}

4 October 2006

\begin{abstract}
We consider a model with an $A_{4}$ flavour symmetry, recently proposed by E. Ma [1, and make a numerical study, through scatter plots, of its neutrino mass matrix.
\end{abstract}

The neutrino mass matrix $\mathcal{M}_{\nu}$ is defined by the neutrino mass term

$$
\mathcal{L}_{\nu \text { mass }}=\frac{1}{2} \nu_{L}^{T} C^{-1} \mathcal{M}_{\nu} \nu_{L}+\text { H.c. },
$$

where $C$ is the Dirac-Pauli charge-conjugation matrix and $\nu_{L}=\left(\nu_{e L}, \nu_{\mu L}, \nu_{\tau L}\right)^{T}$ is the column vector of the three weak-eigenstate left-handed neutrino fields. The matrix $\mathcal{M}_{\nu}$ is symmetric and is diagonalized as

$$
U^{T} \mathcal{M}_{\nu} U=\operatorname{diag}\left(m_{1}, m_{2}, m_{3}\right),
$$

where the lepton mixing matrix $U$ is unitary and the neutrino masses $m_{1,2,3}$ are real and non-negative. The matrix $U$ may be parametrized as

$$
U=P_{F}\left(\begin{array}{ccc}
c_{13} c_{12} & c_{13} s_{12} & s_{13} e^{-i \delta} \\
-c_{23} s_{12}-s_{23} s_{13} c_{12} e^{i \delta} & c_{23} c_{12}-s_{23} s_{13} s_{12} e^{i \delta} & s_{23} c_{13} \\
-s_{23} s_{12}+c_{23} s_{13} c_{12} e^{i \delta} & s_{23} c_{12}+c_{23} s_{13} s_{12} e^{i \delta} & -c_{23} c_{13}
\end{array}\right) P_{M},
$$

\footnotetext{
${ }^{\ddagger}$ E-mail: balio@cftp.ist.utl.pt

§E-mail: helmut.kuehboeck@gmx.at
} 
where

$$
\begin{aligned}
& P_{F}=\operatorname{diag}\left(e^{i \vartheta_{e}}, e^{i \vartheta_{\mu}}, e^{i \vartheta_{\tau}}\right), \\
& P_{M}=\operatorname{diag}\left(e^{i \Theta / 2}, 1, e^{i \Omega / 2}\right)
\end{aligned}
$$

are diagonal unitary matrices. In equation (3),$c_{i j}=\cos \theta_{i j}$ and $s_{i j}=\sin \theta_{i j}$ for $i j=$ $23,13,12$. There are nine observables: the three masses $m_{1,2,3}$, the three mixing angles $\theta_{23,13,12}$, the Dirac phase $\delta$, and the Majorana phases $\Theta$ and $\Omega .{ }^{1}$ Correspondingly, the matrix $\mathcal{M}_{\nu}$ has in general nine parameters: the moduli of its six independent matrix elements, and three phases invariant under $\mathcal{M}_{\nu} \rightarrow X \mathcal{M}_{\nu} X$, where $X$ is an arbitrary diagonal unitary matrix.

Recently [1], E. Ma proposed a model, based on the seesaw mechanism and on a $A_{4}$ symmetry group, wherein $\mathcal{M}_{\nu}$ may be parametrized as

$$
\mathcal{M}_{\nu}=\left(\begin{array}{ccc}
f a^{2} & a b & a c \\
a b & f b^{2} & b c \\
a c & b c & f c^{2}
\end{array}\right)
$$

where $f$ is dimensionless while $a, b$, and $c$ have dimension $M^{1 / 2}$. We may, without loss of generality, assume $a, b$, and $c$ to be real and non-negative: their phases may be absorbed through appropriate rephasings of the $\nu_{\alpha L}$. Ma's model is interesting since it has the potential to be quite predictive: it has only five parameters - $a, b, c$, and the modulus and phase of $f$. This model has as many parameters as the two-texture-zero models of Frampton, Glashow, and Marfatia 2] and of Lavoura [3. It is also remarkable that the form of the mass matrix (6) is invariant under the renormalization-group evolution

$$
\mathcal{M}_{\nu}(\mu)=I\left(\mu, \mu_{0}\right) \mathcal{M}_{\nu}\left(\mu_{0}\right) I\left(\mu, \mu_{0}\right)
$$

where $I\left(\mu, \mu_{0}\right)$ is a diagonal matrix; indeed, the diagonal matrix elements of $I\left(\mu, \mu_{0}\right)$ may be absorbed through redefinitions of $a, b$, and $c$ while keeping the form of $\mathcal{M}_{\nu}$ in (6) unchanged.

When $b=c$ the matrix (6) becomes $\mu-\tau$ symmetric, leading therefore to $\theta_{23}=\pi / 4$ and $\theta_{13}=0$. This is allowed by the experimental data - see below. But, it is important to study in detail the model (6) for $\mathcal{M}_{\nu}$ in order to see how far away from that limiting case $\theta_{23}=\pi / 4, \theta_{13}=0$ that model allows one to go. That's the study that we undertake in this letter. Since it seems difficult to proceed analytically, we have done our study in a purely numerical fashion, by constructing appropriate scatter plots.

The mass matrix (6) is characterized by the relations

$$
\begin{aligned}
& \frac{\left(\mathcal{M}_{\nu}\right)_{e e}}{\left(\mathcal{M}_{\nu}\right)_{\mu \mu}}=\left[\frac{\left(\mathcal{M}_{\nu}\right)_{e \tau}}{\left(\mathcal{M}_{\nu}\right)_{\mu \tau}}\right]^{2}, \\
& \frac{\left(\mathcal{M}_{\nu}\right)_{e e}}{\left(\mathcal{M}_{\nu}\right)_{\tau \tau}}=\left[\frac{\left(\mathcal{M}_{\nu}\right)_{e \mu}}{\left(\mathcal{M}_{\nu}\right)_{\mu \tau}}\right]^{2},
\end{aligned}
$$

\footnotetext{
${ }^{1}$ The phases $\vartheta_{\alpha}(\alpha=e, \mu, \tau)$ are not observable since they may be eliminated through a rephasing of the charged-lepton fields.
} 
which should be compared to the analogous "scaling" relations, recently suggested by Mohapatra and Rodejohann (MR),

$$
\frac{\left(\mathcal{M}_{\nu}\right)_{e \mu}}{\left(\mathcal{M}_{\nu}\right)_{e \tau}}=\frac{\left(\mathcal{M}_{\nu}\right)_{\mu \mu}}{\left(\mathcal{M}_{\nu}\right)_{\mu \tau}}=\frac{\left(\mathcal{M}_{\nu}\right)_{\mu \tau}}{\left(\mathcal{M}_{\nu}\right)_{\tau \tau}}
$$

Both the scaling relations (8) and (9) are invariant under the renormalization-group evolution, and both lead to mass matrices parametrized by four moduli and one phase. However, while the MR scaling relations ${ }^{2}$ lead to the clear-cut predictions $m_{3}=0$ and $\theta_{13}=0,{ }^{3}$ we shall find in the present letter that the scaling relations (8) lead to much less well-defined predictions, largely because of the experimental indeterminacy of the Majorana phases $\Theta$ and $\Omega$.

We have used in our numerical study the following data on the mixing angles:

$$
0.30<\tan ^{2} \theta_{12}<0.61
$$

and

$$
\begin{aligned}
\cos ^{2} 2 \theta_{23} & <0.10 \\
\sin ^{2} \theta_{13} & <0.047 .
\end{aligned}
$$

These are the $3 \sigma$ bounds derived in [7] from the data. Notice that, experimentally, there is no preferred value for $\sin ^{2} \theta_{13}$ : there is only an upper bound on it. As for $\theta_{23}$, its experimentally preferred value is $\pi / 4$ and it is equally probable that it is higher or lower than $\pi / 4$; for this reason, $\cos ^{2} 2 \theta_{23}$ seems to be the appropriate observable. For the neutrino masses we have taken - see [7]-

$$
0.022<\frac{m_{2}^{2}-m_{1}^{2}}{\left|m_{3}^{2}-m_{1}^{2}\right|}<0.065
$$

The sign of $m_{3}-m_{1}$ is unknown; mass spectra with $m_{3}>m_{1}$ are called "normal", those with $m_{3}<m_{1}$ are dubbed "inverted".

We have made extensive scans of the space parametrized by $b / a, c / b,|f|$, and $\arg f$. Whenever the resulting observables agreed with the data (10)-(13), we would fix the overall scale of the neutrino masses by using

$$
m_{2}^{2}-m_{1}^{2}=8.1 \times 10^{-5} \mathrm{eV}^{2} .
$$

Our results are given by scatter plots $1-8$. We have found, numerically, that a normal mass spectrum is obtained when $c \geq b>a$, and an inverted one when $c \leq b<a$. In both cases, good fits of the observables are only obtained when $\arg f$ is very close or equal to $\pi$.

Our main plots are 1 and 2 , in which one sees $\sin ^{2} \theta_{13}$ plotted against $\cos ^{2} 2 \theta_{23}$. The situation $\sin ^{2} \theta_{13}=\cos ^{2} 2 \theta_{23}=0$ is always allowed, since it corresponds to $b=c$. In general,

\footnotetext{
${ }^{2}$ Models leading to the MR scaling relations had already been proposed before the MR paper [5, 6].

${ }^{3}$ This is equivalent to four predictions, since $m_{3}=0$ implies that the Majorana phase $\Omega$ is meaningless, and $\theta_{13}=0$ implies that the Dirac phase $\delta$ is meaningless.
} 
any value of $\cos ^{2} 2 \theta_{23}$ obeying the bound (11), and of $\sin ^{2} \theta_{13}$ obeying the bound (12), is allowed by Ma's model; none of these observables may be zero unless the other one vanishes too. One also sees in figures 1 and 2 that the model generally predicts

$$
\sin ^{2} \theta_{13} \lesssim \frac{\cos ^{2} 2 \theta_{23}}{2} .
$$

When the neutrino mass spectrum is inverted, there is also a distinctive bound

$$
\sin ^{2} \theta_{13} \gtrsim \frac{15 \cos ^{2} 2 \theta_{23}}{100} \quad \text { (inverted spectrum). }
$$

We gather from plots 1 and 2 that, in the case of a normal spectrum, a larger area of the $\cos ^{2} 2 \theta_{23}-\sin ^{2} \theta_{13}$ plane is allowed than in the case of an inverted spectrum. Ma's model seems to prefer in general a normal neutrino mass spectrum. Indeed, while in that case any value for $\tan ^{2} \theta_{12}$ in the range (10) is allowed, when the neutrino mass spectrum is inverted $\tan ^{2} \theta_{12}$ cannot be smaller than 0.5 . This is depicted in plots 3 and 4 , respectively.

Almost-degenerate neutrinos (the situation where $m_{1}^{2} \gg\left|m_{3}^{2}-m_{1}^{2}\right|$ ) are allowed both with normal and inverted mass spectra. Consulting the plots 5 and 6 , we see that, in the case of a normal neutrino mass spectrum, the lowest-mass neutrino may have an almost vanishing mass, while, when the mass spectrum is inverted, the lowest neutrino mass must always be larger than $10^{-2} \mathrm{eV}$.

Experiments on neutrinoless double-beta decay indirectly probe the modulus of the ee matrix element of $\mathcal{M}_{\nu}$, which we denote $\langle m\rangle_{e e}$. This quantity is in Ma's model distinctly correlated with the neutrino masses; we see in plots 7 and 8 that $\langle m\rangle_{e e}$ is always extremely close to one third the average mass of the neutrinos. This is due to the fact that good fits of the experimental observables are in general only obtained when the ratios $b / a$ and $c / b$ are both very close to 1 .

Summarizing, we have analyzed numerically a neutrino mass matrix which may be enforced through a renormalizable model, has few parameters, and is invariant under the renormalization-group evolution. We have found that that neutrino mass matrix has less predictive power than initially guessed. It is able to fit both normal and inverted neutrino mass spectra, though in the latter case only if $\tan ^{2} \theta_{12} \geq 0.5$. The mass responsible for neutrinoless double-beta decay is always very close to one-third the average neutrino mass. The small parameters $\sin ^{2} \theta_{13}$ and $\cos ^{2} 2 \theta_{23}$ may take any value in their experimentally allowed ranges, but they always obey the bound (15), ans also the bound (16) if the neutrino mass spectrum is inverted.

Acknowledgements: We thank Walter Grimus for reading the manuscript and making many useful suggestions. The work of L.L. was supported by the Portuguese Fundação para a Ciência e a Tecnologia through the projects POCTI/FNU/37449/2001 and U777Plurianual. 


\section{References}

[1] E. Ma, Tetrahedral family symmetry and the neutrino mixing matrix, Mod. Phys. Lett. A20 (2005) 2601 hep-ph/0508099.

[2] P.H. Frampton, S.L. Glashow, and D. Marfatia, Zeroes of the neutrino mass matrix, Phys. Lett. B536 (2002) 79 hep-ph/0201008.

[3] L. Lavoura, Zeros of the inverted neutrino mass matrix, Phys. Lett. B609 (2005) 317 hep-ph/0411232.

[4] R.N. Mohapatra and W. Rodejohann, Scaling in the neutrino mass matrix, hep-ph/0608111.

[5] L. Lavoura, New model for the neutrino mass matrix, Phys. Rev. D62 (2000) 093011 hep-ph/0005321;

T. Kitabayashi and M. Yasuè, Neutrino oscillations induced by two-loop radiative mechanism, Phys. Lett. B490 (2000) 236 hep-ph/0006014.

[6] W. Grimus and L. Lavoura, Softly broken lepton number $L_{e}-L_{\mu}-L_{\tau}$ with nonmaximal solar neutrino mixing, J. Phys. G (Particles and Fields) 31 (2005) 683 hep-ph/0410279; Seesaw model with softly broken $L_{e}-L_{\mu}-L_{\tau}$, JHEP 09 (2000) 007 hep-ph/0008020.

[7] M. Maltoni, T. Schwetz, M. Tórtola, and J.W.F. Valle, Status of global fits to neutrino oscillations, New. J. Phys. 6 (2004) 122 hep-ph/0405172. 


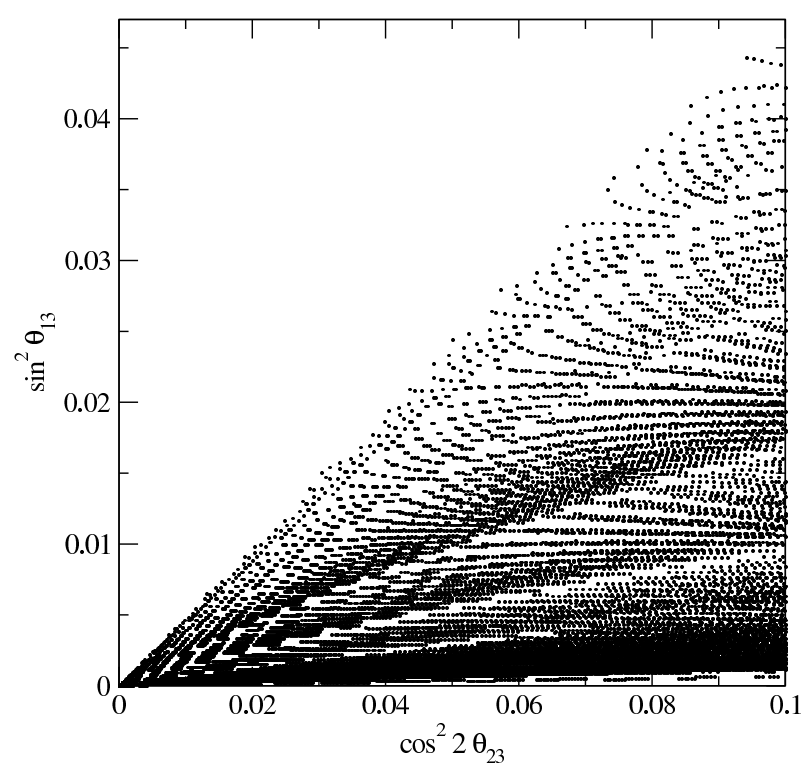

Figure 1: Scatter plot of $\sin ^{2} \theta_{13}$ as a function of $\cos ^{2} 2 \theta_{23}$ in the case of a normal neutrino mass spectrum. 


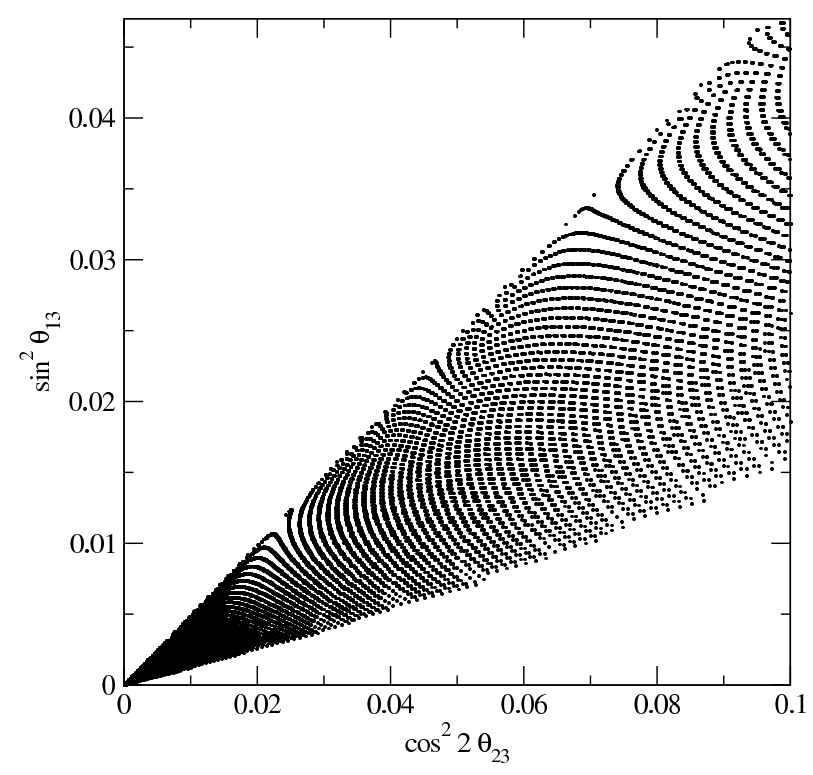

Figure 2: The same as figure 1, but in the case of an inverted neutrino mass spectrum.

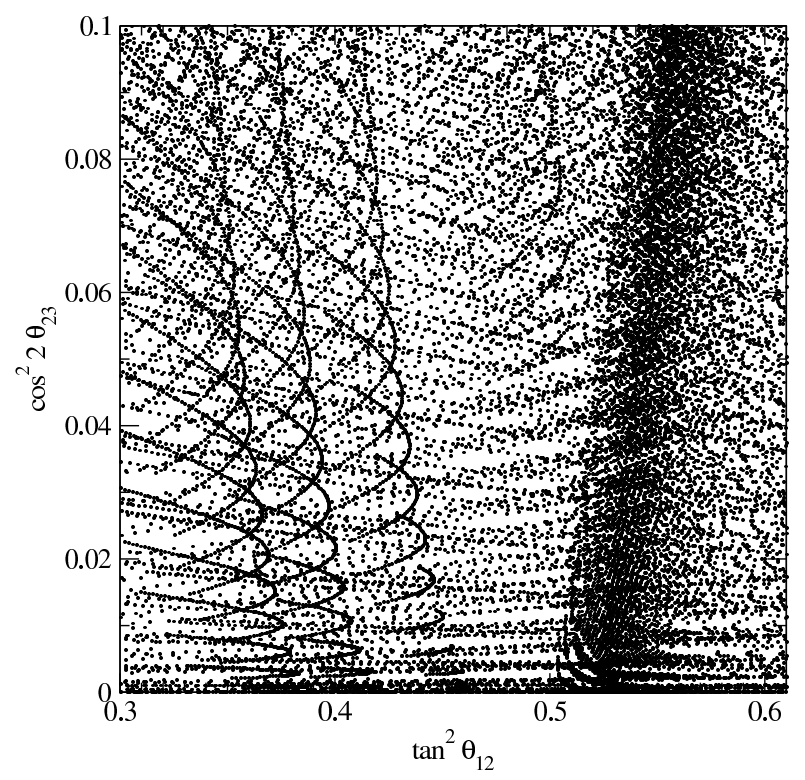

Figure 3: Scatter plot of $\cos ^{2} 2 \theta_{23}$ as a function of $\tan ^{2} \theta_{12}$ in the case of a normal neutrino mass spectrum. 


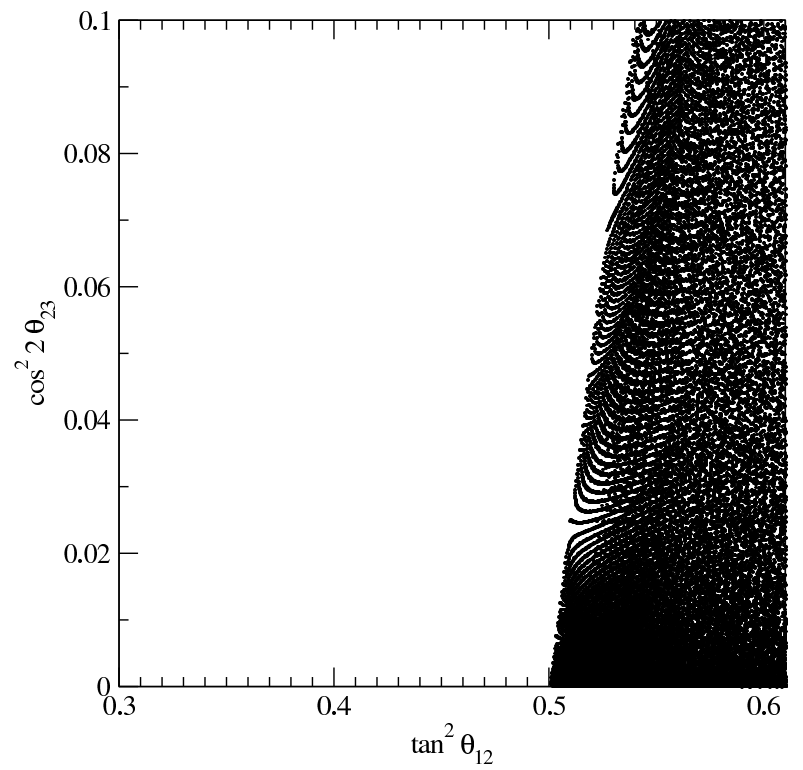

Figure 4: The same as figure 3, but in the case of an inverted neutrino mass spectrum.

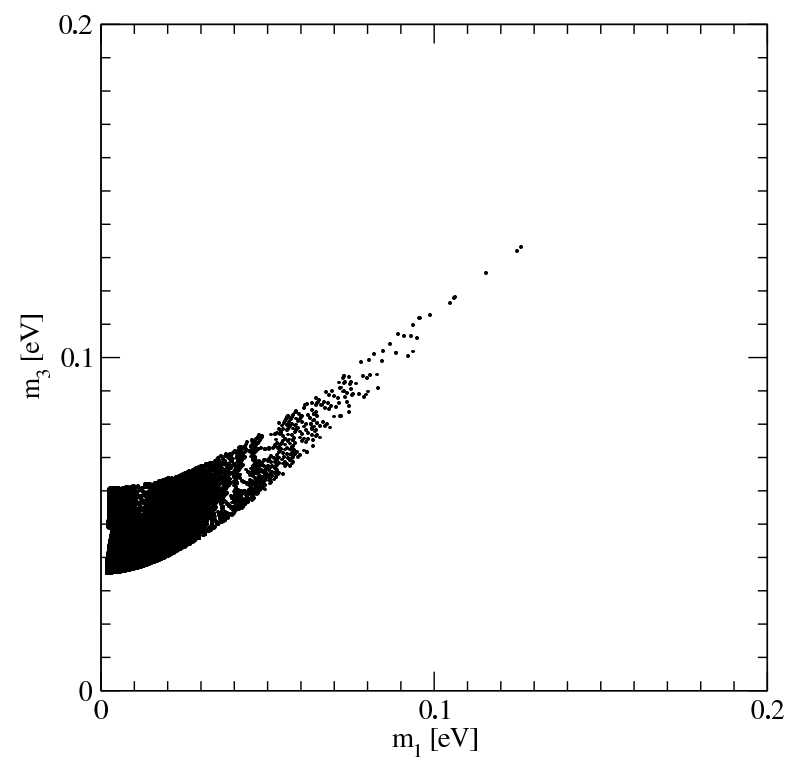

Figure 5: Scatter plot of $m_{3}$ against $m_{1}$ in the case of a normal neutrino mass spectrum. 


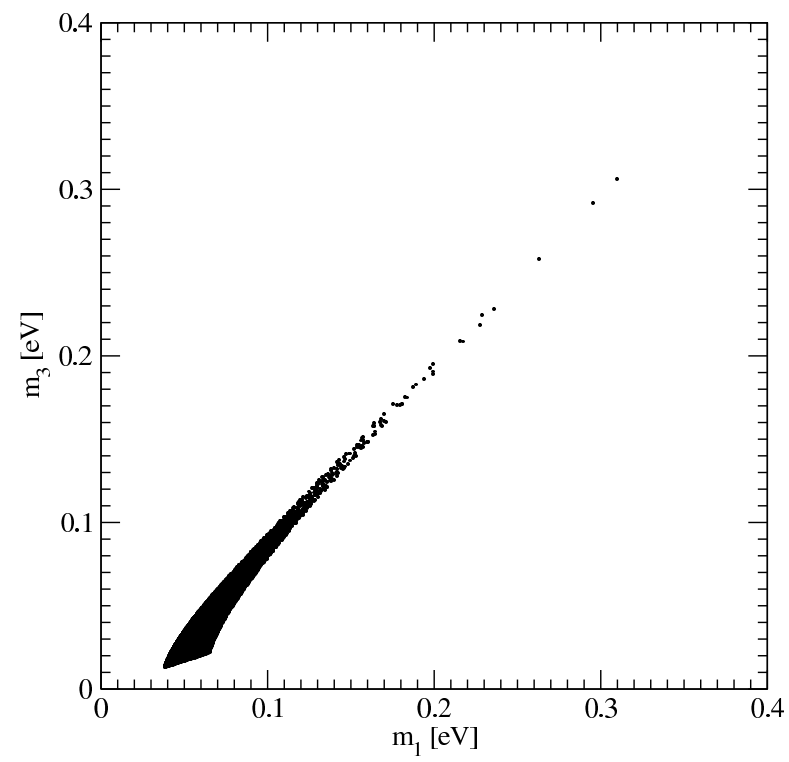

Figure 6: The same as figure 5, but in the case of an inverted neutrino mass spectrum.

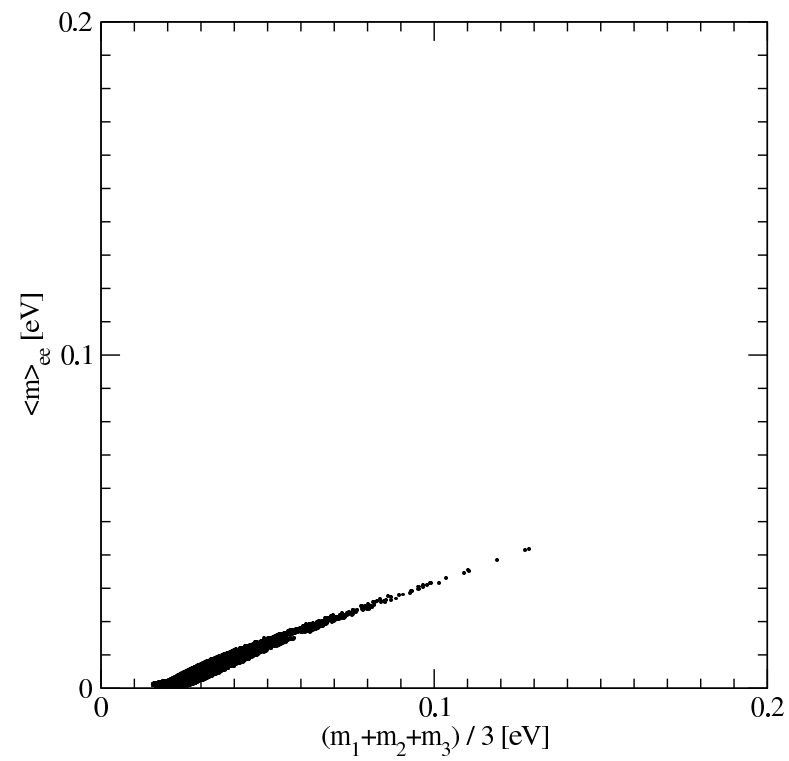

Figure 7: The mass relevant for neutrinoless double-beta decay as a function of the average mass of the neutrinos in the case of a normal neutrino mass spectrum. 


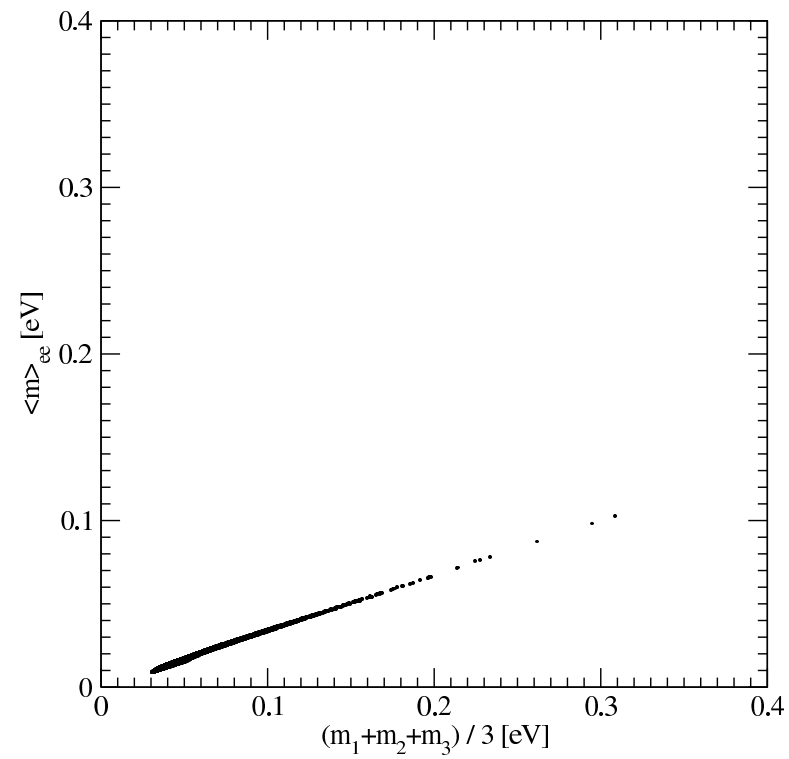

Figure 8: The same as figure 7, but in the case of an inverted neutrino mass spectrum. 